\title{
DISTRIBUTIONS DE PARETO: INTÉRÊTS ET LIMITES EN RÉASSURANCE
}

\author{
Par Claude Huyghues-Beaufond \\ Assurances Générales de France, Paris, France
}

\begin{abstract}
Instead of determining for a fire insurance portfolio the loss distribution purely based on the claims experience, we try to determine it based on the sums insured.
\end{abstract}

\section{KEYWORDS}

Loss distribution based on sums insured; Pareto distribution.

\section{INTRODUCTION}

Au lieu d'estimer, sur la base unique des sinistres, leur loi de distribution, on va essayer de le faire sur la base du profil du portefeuille qui les génère.

En Assurances Décès, s'il n'y a pas de relation entre le capital garanti en cas de décès et l'âge de l'assuré, les sinistres sont distribués comme le sont les capitaux assurés.

En Assurance Dommages, ce n'est généralement pas le cas.

Par exemple, si les risques assurés d'une compagnie sont distribués sur une loi de Pareto tronquée au plein de souscription, que la distribution des taux de dommages vérifie certaines propriétés acceptables, alors les sinitres ne sont pas issus d'une distribution tronquée de Pareto.

\section{CADRE DE NOTRE ÉTUDE}

Les hypothèses utilisées sont celles implictement admises pas les réassureurs:

- Le portefeuille assuré comprend $N$ risques $\left(R_{i}\right)_{i=1, \ldots N}$ de valeurs assurées $\left(K_{i}\right)_{i=1 \ldots N}$.

- Le portefeuille est stable dans le temps sur les $m$ dernières années (nombre de polices par tranche de capitaux assurés) et pour l'année $m+1$.

- Pendant chacune de ces dernières années, et pour l'année $m+1$, pour chacun des $N$ risques:

- Si $\left(s i_{j}\right)_{i=1 \ldots n i}$ désignent les $n_{i}$ sinistres sur le risque $i, p\left(n_{i}=o\right)=$ $\exp \left(-p_{i}\right)=\exp (-p)$

- $\forall t$ dans $[0,1], \forall i, \forall j, p\left[\left(s i_{j} / K_{i}\right) \leq t\right]=T(t)$ ne dépend pas de $i$ et de $j$. 
- $T$ est une fonction continue sur $[0,1]$, dérivable sur $[0,1]$ strictement croissante sur $[0,1]$

- $n_{i}$ suit une loi de Poisson de paramètre $p$.

- Les sinitres survenant sont indépendants les uns des autres.

$N . B .:$ Les hypothèses sur la fonction de distribution $T$ de taux de dommages et sur la fréquence $p$ sont assez restrictives.

On pourrait, sans trop de difficultés, les rendre moins contraignantes en écrivant que les risques $i$ souscrits se caractérisent ainsi: ils sont issus d'une communauté $M$ de risques $I$ pour lesquels nous avons:

1. $\forall_{I}, n_{I}$ suit une loi de Poisson de paramètre $p_{I}$.

2. $\forall_{I}, \forall_{j}, \forall_{t}, p\left(\left[s_{I j} / K_{I}\right] \leq t\right)=T_{I}(t)$ ne dépend que de $I$.

3. Il n'y a pas de corrélation entre $p_{I}$ et $K_{I}$, d'une part, entre $T_{I}$ et $K_{I}$ d'autre part, ou encore:

$\forall(a, b) a<b$,

$E_{M}\left(p_{I} /\left[K_{I} \in[a, b]\right]\right)=p$ ne dépend pas de $[a, b]$

$E_{M}\left(T_{I}(t) /\left[K_{I} \in[a, b]\right]\right)=T(t)$ ne dépend pas de $[a, b]$

Alors, toutes les espérances écrites par la suite subsistent; elles sont des espérances d'espérances conditionnelles.

Les contraintes ainsi exposées n'altèrent en rien la latitude d'acceptation de l'assureur ni son niveau d'acceptation, notamment lorsqu'il y a beaucoup de coassurance.

\section{PROBLÈME}

On cherche à déterminer la loi $L_{x}$ du nombre de sinistres supérieurs à $x$.

Montrons, dans un premier temps, que $L_{x}$ est une loi de Poisson dont il suffira de connaître la moyenne $\bar{L}_{x}$ (E. STraub, 1971).

- Pour un risque $i$,

$L_{i x}$ suit une loi de Poisson de paramètre $p\left[1-T\left[\min \left(1, \frac{x}{K_{i}}\right)\right]\right]$

- C'est évident si $x>K i$.

- Si $x \leq K i$, alors:

$$
\begin{aligned}
\left.p\left[L_{i x}=n\right)\right] & =\sum_{j=n}^{+\infty}\left(\begin{array}{l}
j \\
n
\end{array}\right) \exp (-p) \frac{p^{j}}{j !} T\left(\frac{x}{K_{i}}\right)^{j-n}\left[1-T\left(\frac{x}{K_{i}}\right)\right]^{n} \\
& =\exp (-p) \frac{p^{n}}{n !}\left[1-T\left(\frac{x}{K_{i}}\right)\right]^{n} \sum_{j=n}^{\infty} \frac{p^{j-n}}{(j-n) !} T\left(\frac{x}{K_{i}}\right)^{j-n}
\end{aligned}
$$




$$
\begin{aligned}
& \left.\left.=\exp (-p) \exp \left[p T\left(\frac{x}{K_{i}}\right)\right]\right] p\left[1-T\left(\frac{x}{K_{i}}\right)\right]\right]^{n} \frac{1}{n !} \\
& =\exp \left[-\left[p\left(1-T\left(\frac{x}{K_{i}}\right)\right)\right]\right]\left[p\left[1-T\left(\frac{x}{K_{i}}\right)\right]\right]^{n} \frac{1}{n !}
\end{aligned}
$$

- Pour chaque risque $i$,

$L_{i x}$ suit une loi de Poisson de paramètre $p\left[1-T\left[\min \left(1, \frac{x}{K_{i}}\right)\right]\right]$

- $L_{x}=\sum_{i} L_{i x}$. Les $L_{i x}$ étant indépendantes,

- $L_{x}$ suit une loi de Poisson de paramètre

$$
p \sum_{1}\left[1-T\left[\min \left(1, \frac{x}{K_{i}}\right)\right]\right]=\bar{L}_{x}=L(x)
$$

\section{L'APPROCHE DES RÉASSUREURS}

Pour évaluer le coût d'une couverture en excédent de sinistres, l'assureur et le réassureur ont besoin de connaître $L(x)$, l'espérance mathématique du nombre de sinistres supérieurs à $x$. Pour cela, on va passer du cas discret évoqué précédemment au cas continu.

Ainsi, la prime pure requise pour une couverture $A x s B$ est-elle:

$$
P=A L(A+B)-\int_{B}^{A+B}(x-B) \frac{d L(x)}{d x} d x
$$

- Distribution des capitaux assurés :

- On supposera que les risques sont distribués en montant, sur une loi de Pareto de paramètre $\alpha$ pour $K>c, c$ étant fixé. On montrera, sur un exemple en annexe, que cette hypothèse est acceptable.

De la connaissance de la valeur certaine $N_{c}$, nombre de risques supérieurs à $c$, on estime $N_{x}$ :

$$
\hat{N}_{x}=N_{c}\left(\frac{c}{x}\right)^{\alpha}
$$

Si, lors de l'estimation, $\hat{\alpha}$ est non biaisé, alors $\hat{N}_{x}$ surestime en moyenne $N_{x}$.

En effet, $\hat{N}_{x}=N_{x}\left(\frac{c}{x}\right)^{\varepsilon}$ avec $E(\varepsilon)=0$. 


$$
\left.E\left[\left(\frac{c}{x}\right)^{\varepsilon}\right]=E\left(\exp \left(\varepsilon \ln \frac{c}{x}\right)\right) \geq \exp \left[E\left(\varepsilon \ln \left(\frac{c}{x}\right)\right)\right]=1\right]
$$

- Calcul de $L(x)$ :

$$
\begin{aligned}
L(x) & =\int_{x}^{+\infty}-\frac{d N_{u}}{d u} p\left[1-T\left(\frac{x}{u}\right)\right] d u \\
& =\int_{x}^{+\infty} \alpha N_{c} \frac{c^{\alpha}}{u^{\alpha+1}} p\left[1-T\left(\frac{x}{u}\right)\right] d u \\
& =\int_{x}^{+\infty} N_{c} \frac{c^{\alpha}}{u^{\alpha+2}} p x t\left(\begin{array}{c}
x \\
- \\
u
\end{array}\right) d u
\end{aligned}
$$

après intégration par partie.

Par ailleurs $L^{\prime}(x)=-\alpha N_{c} \frac{c \alpha}{x^{\alpha+1}} p[1-T(1)]-\int_{x}^{+\infty} p \alpha N_{c} \frac{c^{\alpha}}{u^{\alpha+2}} t\left(\begin{array}{c}x \\ u\end{array}\right) d u$ soit $L^{\prime}(x)=-\frac{\alpha}{x} L(x)$ ou encore $L(x)=x^{-\alpha} a^{\alpha} L(a), \forall a \geq c$ ( $t$ désigne la dérivée de $T$ ).

On rappelle ici que, si $F(x)=\int_{c}^{x} f(x, u) d u$, et que la différentielle $d f \operatorname{de} f$ existe, alors $F^{\prime}$ existe et $F^{\prime}(x)=f(x, x)+\int_{c}^{x} \frac{\partial f}{\partial x}(x, u) d u$

- Intérêt d'une telle formule:

$T$ et $p$ existent mais sont inconnus. On va revenir à l'expérience du passé et estimer:

$L(a) \operatorname{par} \hat{L}(a)=\frac{\text { nombre de sinistres survenus supérieurs à } a \text { en } m \text { années }}{m}$

$$
=\frac{m_{a}}{m}
$$

pour tout $a \geq c$.

On choisit $a$ le plus faible possible pour minimiser $\frac{\sigma[L(y)]}{L(y)}=\frac{1}{\sqrt{L}(y)}$ On prend donc $a=c$. 
En effet, l'erreur relative sur $L(x)$ est d'autant plus faible qu'est faible l'erreur relative sur $L(a)$.

$(\sigma[L(y)]$ désigne l'écart type de $L y)$.

- Tarification :

- Fréquence de sinistres supérieurs à $B$ :

$\hat{f}_{B}=\hat{f}_{c}\left(\frac{c}{B}\right)^{\alpha}$

- Coût moyen d'un sinistre $d^{\prime} x s$.

Coût moyen d'un sinistre supérieur à $B$ : pour $\alpha>1$

$$
\begin{aligned}
c_{m}(B) & =\int_{B}^{+\infty}(x-B) d F_{\alpha}(x) /\left[1-F_{\alpha}(B)\right] \\
& =\int_{B}^{+\infty}\left[1-F_{\alpha}(x)\right] d x /\left[1-F_{\alpha}(B)\right] \\
& =\int_{B}^{+\infty} \frac{x^{-\alpha}}{a^{-\alpha}} d x /\left[1-F_{\alpha}(B)\right]=\frac{B}{\alpha-1}
\end{aligned}
$$

-- Coût moyen d'un sinistre $d^{\prime} x s$ :

$$
\begin{aligned}
c_{m}(A, B) & =c_{m}(B)-\frac{1-F_{\alpha}(A+B)}{1-F_{\alpha}(B)} c_{m}(A+B) \\
& =\frac{B}{\alpha-1}-\frac{A+B}{\alpha-1}\left(\frac{B}{A+B}\right)^{\alpha}
\end{aligned}
$$

- Estimation de la prime pure requise:

$$
\hat{P}=\frac{m_{c}}{m}\left(\frac{c}{B}\right)^{\alpha} \frac{B}{\alpha-1}\left[1-\left(\frac{B}{A+B}\right)^{\alpha-1}\right]
$$

Cette formule reste vraie pour $0<\alpha<1$.

Il suffit d'écrire

$$
\left[1-F_{\alpha}(B)\right] C_{m}(A, B)=\int_{B}^{A+B}(x-B) d F_{\alpha}(x)+A\left[1-F_{\alpha}(A+B)\right]
$$

Pour $\alpha=1$ 
$P=\frac{m_{c}}{m} \frac{c}{B} B \lim _{\varepsilon \rightarrow 0} \frac{1}{\varepsilon}\left[1-\left(\frac{B}{A+B}\right)^{\varepsilon}\right]=\frac{m_{c}}{m} c \ln \left(\frac{B+A}{B}\right)$

Le grand avantage de cette formulation réside dans la disparition de $p$ et de $T$.

Le réassureur ne peut estimer $p$ car il ne connaît que les sinistres supérieurs à un certain seuil $s$, qu'on a supposé ici inférieur à $c$. Pour les mêmes raisons, il ne peut estimer $T$.

A fortiori, dans l'hypothèse d'hétérogénéité, on ne peut estimer $p_{i}$ et $T_{i}$ pour chaque $i$, d'autant plus qu'on ne connaît pas les fonctions de structures de $(p)$ et $(T)$.

\section{CRITIQUE DE LA MÉTHODE}

On a supposé que $T$ existait, mais était inconnue (si $T$ était connu, $L(x)$ le serait immédiatement.

Des fonctions $T$ circulent chez les réassureurs, sans qu'ils sachent bien lesquelles utiliser.

La méthode précédemment décrite a l'énorme avantage de ne pas requérir la connaissance de $T$.

Malheureusement, elle présente un énorme défaut. $L(x)$ est calculé en écrivant $N_{x}=N_{c}\left(\frac{c}{x}\right)^{\alpha}$ pour tout $x$ et même si $x$ est supérieur à $F$, le plein de souscription.

Or $N_{y}=o$ pour $y>F$. Il nous faut donc, au mieux, utiliser une loi de Pareto tronquée au point $F$. On supposera donc que toute la densité de risques supérieurs à $F$, suivant l'hypothèse paretienne, est concentrée en $F$, après dégagement par exemple en Facultatives proportionnelles.

$$
\text { Ainsi, } \begin{aligned}
L(y) & =\int_{y}^{F} \frac{-d N_{u}}{d u} p\left[1-T\left(\frac{y}{u}\right)\right] d u+N_{c}\left(\frac{c}{F}\right)^{\alpha} p\left[1-T\left(\frac{y}{F}\right)\right] \\
& = \\
\downarrow & L_{1}(y) \quad+\quad L_{2}(y)
\end{aligned}
$$

En intégrant par partie,

$$
\begin{aligned}
L_{1}(y) & =-N_{F} p\left[1-T\left(\frac{y}{F}\right)\right]+\int_{y}^{F} y N_{u} / u^{2} p t\left(\frac{y}{u}\right) d u \\
L_{1}^{\prime}(y) & =\frac{d N_{u}}{d u}(y) p\left[1-T\left(\frac{y}{y}\right)\right]+\int_{y}^{F} \frac{d N_{u}}{d u} \frac{p}{u} t(y / u) d u \\
& =-\alpha \int_{y}^{F} p \frac{N_{u}}{u^{2}} t\left(\frac{y}{u}\right) d u
\end{aligned}
$$


soit $\frac{y}{\alpha} L_{1}^{\prime}(y)=-L_{1}(y)-N_{c}\left(\frac{c}{F}\right)^{\alpha} p\left[1-T\left(\frac{y}{F}\right)\right]$

En posant $L_{1}(y)=\phi(y) y^{-\alpha} N c\left(\frac{c}{F}\right)^{\alpha} p$

$L_{1}^{\prime}(y)=\frac{\phi^{\prime}(y)}{\phi(y)} L_{1}(y)-\frac{\alpha}{y} L_{1}(y)$

soit $\phi^{\prime}(y)=-\alpha y^{\alpha-1}\left[1-T\left(\frac{y}{F}\right)\right]$

soit $\phi(y)=\phi(F)+\alpha \int_{y}^{F} u^{\alpha-1}\left[1-T\left(\frac{u}{F}\right)\right] d u$

La condition $\phi(F)=0[\operatorname{car} L(F)=0$ puisque $T(u)=1 \Rightarrow u=1]$ nous indique que :

$L_{1}(y)=\alpha\left[\int_{y}^{F} u^{\alpha-1}\left[1-T\left(\frac{u}{F}\right)\right] d u\right] y^{-\alpha} N_{c}\left(\frac{c}{F}\right)^{\alpha} p$

et

$L(y)=\left[\left[\int_{y}^{F} u^{\alpha-1}\left[1-T\left(\frac{u}{F}\right)\right] d u\right] \alpha y^{-\alpha}+\left[1-T\left(\frac{y}{F}\right)\right]\right] N_{c}\left(\frac{\mathrm{c}}{\mathrm{F}}\right)^{\alpha} p$

\section{Exemple: Prenons}

$-\alpha=2$ pour $x>1$ M FRF.

$-t(u)=1$ sur $[0,1]$

$\therefore p=1 \%$; 10.000 risques de valeurs assurées $>1 \mathrm{M} \mathrm{FRF}$.

$-F=10$ M FRF.

$$
\begin{aligned}
L_{1}(y) & =-\int_{y}^{F} p \frac{d}{d u}\left[10.000\left(\left(\frac{10}{u}\right)^{6}\right)^{2}\right]\left(1-\frac{y}{u}\right) d u \\
& =10^{13}\left[\frac{1}{3 y^{2}}-\frac{1}{F^{2}}+\frac{2 y}{3 F^{3}}\right]
\end{aligned}
$$

On a supposé 10.000 risques $>1 \mathrm{M}$ FRF. Or, on n'en dénombre ici que $9.900=10.000 \times F_{\alpha}(10 \mathrm{M}$ FRF $)$.

Supposons, au pire, que les 100 risques «manquants» se situent en $F$ comme on l'a fait précédemment. 
Alors $L(y)$, espérance du nombre de sinistres supérieurs à $y$, s'écrit:

$$
L(y)=L_{1}(y)+\left[1-F_{\alpha}(F)\right] N_{c} p\left[1-T\left(\frac{y}{F}\right)\right]
$$

Pour notre exemple, $L(y)=L_{1}(y)+0,1\left[1-\frac{y}{F}\right]$

TABLEAU RÉCAPITULATIF

\begin{tabular}{|c|c|c|c|c|}
\hline$x$ & $\begin{array}{l}L(x) \\
(1)\end{array}$ & $\begin{array}{c}L_{1}(x) \\
\text { avec troncation }\end{array}$ & $\begin{array}{l}L(x) \\
(2)\end{array}$ & $(2) /(1)$ \\
\hline 1.000 .000 & 3,3 & 3,2 & 3,3 & $\simeq 100 \%$ \\
\hline 2.000 .000 & 0,83 & 0,74 & 0,82 & $98,7 \%$ \\
\hline 3.000 .000 & 0,37 & 0,29 & 0,36 & $97,3 \%$ \\
\hline 4.000 .000 & 0,21 & 0,14 & 0,20 & $95,2 \%$ \\
\hline 5.000 .000 & 0,13 & 0,06 & 0,11 & $85 \%$ \\
\hline 6.000 .000 & 0,093 & 0,033 & 0,073 & 78 \\
\hline 7.000 .000 & 0,068 & 0,015 & 0,045 & 66 \\
\hline 8.000 .000 & 0,052 & 0,006 & 0,026 & 50 \\
\hline 9.000 .000 & 0,041 & 0,001 & 0,011 & 27 \\
\hline 10.000 .000 & 0,033 & 0 & 0 & 0 \\
\hline
\end{tabular}

La prise en compte de la troncation (2) modifie donc la loi de Pareto des sinistres (1). Les sinistres ne sont alors plus distribués sur une loi de Pareto.

Intuitivement, cela s'explique par le fait qu'il n'y a pas de générations de risques supérieurs à $F$ pour remplacer ceux qui disparaissent (ceux $<x$ ). Cette modification peut être déterminante.

\section{CONCLUSION}

A travers cet exemple, force est de constater que la connaissance d'un profil de portefeuille, d'une espérance de sinistres en nombre à un seuil suffisamment bas pour que l'expérience nous en donne un bon indicateur ne suffisent par à déterminer une espérance (en nombre de sinistres) de sinistres importants qui pourraient affecter lourdement le compte d'exploitation d'un assureur.

La connaissance de la loi de distribution des taux de dommages des riques en portefeuille s'avère nécessaire.

\section{ANNEXE 1}

\section{Estimation de $a$}

$1^{\text {er }}$ cas

Les valeurs assurées des $J$ risques $K_{1}, K_{2}, \ldots K_{J}$ des risques supérieurs à $c$ sont connues. 
La vraisemblance $V\left(K_{1}, K_{2}, \ldots K_{J}, \alpha\right)$ de notre échantillon s'écrit:

$$
V=\prod_{i=1}^{J}\left[\alpha\left(\frac{c}{K_{i}}\right)^{\alpha} x \frac{1}{K_{i}}\right]=\alpha^{J} c^{\alpha J}\left[\prod_{i=1}^{J} K_{i}\right]^{-1-\alpha}
$$

En maximisant le logarithme de $V,\left[\frac{\partial}{\partial \alpha}\left[\ln V\left(\left(K_{i}\right), \hat{\alpha}\right)\right]=0\right]$,

nous obtenons: $\hat{\alpha}=\frac{J}{\sum_{i} \ln \left(\frac{K_{i}}{c}\right)}$

- On peut montrer que $\hat{\alpha}$ est asymptotiquement non biaisé (MetTe RYTGAARD, 1989).

- $\hat{\alpha}$ est une fonction continue de $\left(K_{1}, \ldots K_{J}\right)$.

$2^{\mathrm{e}}$. cas

On ne connaît, en général, que le nombre de polices par tranche de capital assuré, pour les risques supérieurs à $c$.

\begin{tabular}{lcc}
\hline Tranche & $\begin{array}{c}\text { Numéro } \\
\text { de tranche }\end{array}$ & $\begin{array}{c}\text { Nombre } \\
\text { de risques }\end{array}$ \\
\hline$A_{0}-A_{1}$ & 1 & $n_{1}$ \\
\hdashline$A_{i}-A_{i+1}$ & $-1+1$ & $n_{i+1}$ \\
\hline$A m-1-+\infty$ & $--\cdots$ & $-1-$ \\
$\Sigma$ & $m$ & $n_{m}$ \\
\hline
\end{tabular}

On va procéder par étapes pour estimer $\hat{\alpha}$ en utilisant la continuité de $\phi$ : $\left(K_{1}, \ldots K_{J}\right) \rightarrow \hat{\alpha}\left(K_{1}, \ldots K_{J}\right)=\phi\left(K_{1}, \ldots K_{J}\right)$ en utilisant l'estimateur du Khi-deux minimum, par l'algorithme de NEWTON-RAPHSON.

\section{Première étape}

On répartit dans chacune des tranches les risques uniformément.

$\left\{\begin{array}{l}\text { Pour } i<m, \text { les } n_{i} \text { risques valent } B_{i j}=A_{i-1}+\frac{j}{n_{i}}\left(A_{i}-A_{i-1}\right) . \\ \text { Pour } i=m, \text { les } n_{m} \text { risques valent } A_{m-1} .\end{array}\right.$

Pour $j=1 \ldots n_{j}$.

On en déduit $\hat{\alpha}_{1}$, puisqu'on s'est ramené au premier cas. 


\section{Deuxième étape}

On calcule, sous l'hypothèse, $\alpha=\hat{\alpha}_{1}$, les fréquences théoriques de chacune des $m$ tranches de capital assuré.

$$
\text { Pour } j=1 \ldots m, P_{t h_{i}}\left(\hat{\alpha}_{1}\right)=F_{\hat{\alpha}_{1}}\left(A_{i}\right)-F_{\hat{\alpha}_{1}}\left(A_{i-1}\right) \text { avec } \quad \mathrm{A}_{\mathrm{m}}=+\infty
$$

\section{Troisième étape}

On écrit $\hat{\alpha}_{2}=\hat{\alpha}_{1}+\varepsilon$.

$\hat{\alpha}_{2}$ doit être un meilleur estimateur que $\hat{\alpha}_{1} . \varepsilon$ doit être "petit» compte tenu de la continuité de $\phi$.

On écrit alors $P_{t h_{i}}\left(\hat{\alpha}_{2}\right)=P_{t h_{i}}\left(\hat{\alpha}_{1}\right)+\varepsilon \frac{\partial P_{t h_{i}}}{\partial \alpha}\left(\hat{\alpha}_{1}\right)$, au premier ordre.

\section{Quatrième étape}

On cherche à minimiser $G(x)$, avec

$$
\begin{aligned}
G\left(\hat{\alpha}_{2}\right) & =\sum_{i=1}^{m}\left[\left(P_{t h_{i}}\left(\hat{\alpha}_{2}\right)-\frac{n_{i}}{N}\right)^{2} / P_{t h_{i}}\left(\hat{\alpha}_{2}\right)\right] \\
& =A\left(\hat{\alpha}_{1}\right)+\varepsilon B\left(\hat{\alpha}_{1}\right), \text { au premier ordre. }
\end{aligned}
$$

On en déduit: $\hat{\alpha}_{2}=\hat{\alpha}_{1}-\frac{A\left(\hat{\alpha}_{1}\right)}{B\left(\hat{\alpha}_{1}\right)}$

Avec:

$A\left(\hat{\alpha}_{1}\right)=G\left(\hat{\alpha}_{1}\right)$

$B\left(\hat{\alpha}_{1}\right)=\frac{\partial G}{\partial \alpha}\left(\hat{\alpha}_{1}\right)=\sum_{i=1}^{m} \frac{\partial P_{t h_{i}}}{\partial \alpha}\left(\hat{\alpha}_{1}\right) \times\left[2 \frac{\left[P_{t h_{i}}\left(\hat{\alpha}_{1}\right)-\frac{n_{i}}{N}\right]}{P_{t h_{i}}\left(\hat{\alpha}_{1}\right)}-\frac{\left[P_{t h_{i}}\left(\hat{\alpha}_{1}\right)-\frac{n_{i}}{N}\right]^{2}}{P_{t h_{i}}\left(\hat{\alpha}_{1}\right)^{2}}\right]$ et $\quad P_{t h_{i}}(\alpha)=\left(\frac{A_{o}}{A_{i-1}}\right)^{\alpha}-\left(\frac{A_{o}}{A_{i}}\right)^{\alpha}$ et $\frac{\partial P_{t h_{i}}}{\partial \alpha}(\alpha)=\ln \frac{A_{o}}{A_{i-1}} \times\left(\frac{A_{o}}{A_{i-1}}\right)^{\alpha}-\ln \frac{A_{o}}{A_{i}} \times\left(\frac{A_{o}}{A_{i}}\right)^{\alpha}$

On réitère éventuellement le procédé en substituant $\alpha_{2}$ à $\alpha_{1}$ et ainsi de suite. 
Cet algorithme a des chances de converger sous l'hypothèse d'une adéquation parfaite à une loi de Pareto de paramètre $\alpha_{c}$.

En effet:

$$
\begin{aligned}
& G\left(\alpha_{c}\right)=o \\
& G^{\prime}\left(\alpha_{c}\right)=o \\
& G^{\prime \prime}\left(\alpha_{c}\right)=2 \sum_{i=1}^{m}\left[\frac{\partial P_{t h_{i}}}{\alpha_{c}}\left(\alpha_{c}\right)\right]^{2} x \frac{1}{P_{t h(\alpha c)}}>o \\
& \alpha_{n+1}=\alpha_{n}-\frac{G\left(\alpha_{n}\right)}{G^{\prime}\left(\alpha_{n}\right)}=\alpha_{n}-\frac{\left(\alpha_{n}-\alpha_{c}\right)^{2}}{2} \frac{\left[G^{\prime \prime}\left(\alpha_{c}\right)+o_{1}\left(\alpha_{n}-\alpha_{c}\right)\right]}{\left(\alpha_{n}-\alpha_{c}\right)\left[G^{\prime \prime}\left(\alpha_{c}\right)+o_{2}\left(\alpha_{n}-\alpha_{c}\right)\right]} \\
& \text { avec } o_{1}(x) \rightarrow o \text { et } o_{2}(x) \rightarrow o \text { lorsque } x \rightarrow o
\end{aligned}
$$

Nous avons donc:

$\alpha_{n+1}-\alpha_{c}=\left(\alpha_{n}-\alpha_{c}\right)\left[1-\frac{1}{2}+o_{3}\left(\alpha_{n}-\alpha_{c}\right)\right]$ avec $o_{3}(x) \rightarrow o$ lorsque $x \rightarrow o$.

Puisque $1-\frac{1}{2}=\frac{1}{2}$, dans un voisinage de $\alpha_{c},\left|\alpha_{n+1}-\alpha_{c}\right|<\frac{3}{4}\left|\alpha_{n}-\alpha_{c}\right|$ on en déduit que $\alpha_{n} \rightarrow \alpha_{c}$, lorsque $n \rightarrow+\infty$, si $\alpha_{1}$ est suffisamment proche de $\alpha_{c}$.

\section{Application numérique}

Elle se fera sur le portefeuille Incendie (Risque Simples) d'une compagnie d'assurance allemande. On ne considérera ici que les risques supérieurs à $c=5$, l'unité monétaire étant ici occultée.

\begin{tabular}{crc}
\hline Tranche & Nombre de risques \\
\hline $1-$ & 5 & 2.520 \\
$5-$ & 10 & 700 \\
$10-$ & 20 & 514 \\
$20-$ & 50 & 517 \\
$50-100$ & 284 \\
$100-200$ & 200 \\
$200-500$ & 203 \\
$500-1.000$ & 115 \\
$>1.000$ & 289 \\
$\Sigma$ & 5.342 \\
\hline
\end{tabular}




\begin{tabular}{cc}
\hline Nombre d'itérations & $\hat{\alpha}$ \\
\hline 1 & 0,415 \\
2 & 0,392 \\
3 & 0,410 \\
4 & 0,350 \\
5 & 0,380 \\
6 & 0,399 \\
7 & 0,421 \\
8 & 0,403 \\
9 & 0,441 \\
10 & 0,420 \\
11 & 0,401 \\
12 & 0,429 \\
\hline
\end{tabular}

Observons par ailleurs $G(\alpha) N$.

$$
\begin{array}{lll}
G(0,38) N=30,16 & G(0,40) N=10,07 & G(0,42) N=13,21 \\
G(0,39) N=17,15 & G(0,41) N=8,79 & G(0,405) N=8,71 \\
& & G(0,415) N=10,29
\end{array}
$$

Si l'on teste l'hypothèse d'adéquation de la distribution des capitaux assurés à une distribution de Pareto de paramètre $\alpha=0,405$ par la loi du Khi-deux, nous avons $\kappa_{(9-1-1)}^{2}(U)=\kappa_{7}^{2}(U)=95 \%$ implique $U=14,1$

Or $8,71<14,1$; on peut donc accepter l'hypothèse paretienne avec un seuil de tolérance de $5 \%$. Cette méthode du Khi-deux minimum nous donne même le seuil critique, si on le souhaite.

\section{ANNEXE 2}

Il n'est pas toujours évident de trouver un portefeuille, comme celui qu'on vient de décrire, qui se prête à un ajustement des capitaux assurés sur une distribution de Pareto.

La question, ici, est de savoir pourquoi ces lois sont souvent prises en référence.

On part d'un principe bien simple: l'espérance du nombre de sinistres à charge doit être proportionnelle au nombre de risques exposés.

Les lois de Pareto (malgré la réserve importante qu'on a émise précédemment) sont solution.

On a vu, en effet, que $-\frac{L^{\prime}(x)}{L(x)}=-\frac{N_{x}^{\prime}}{N_{x}}=\frac{\alpha}{x}$ soit encore $L(x)=k N_{x}$.

Montrons que ce sont les seules solutions de ce problème, pour toute loi $T(t)$.

On a $L(x)=d N_{x}$ soit $\frac{L^{\prime}(x)}{L(x)}=\frac{N_{x}^{\prime}}{N_{x}}$ 


$$
\text { Comme } \begin{aligned}
L(x) & =\int_{x}^{+\infty}-\frac{d N_{u}}{d_{u}}\left[1-T\left(\frac{x}{u}\right)\right] d u \\
& =\int_{x}^{+\infty} N_{u} \frac{x}{u^{2}} t\left(\frac{x}{u}\right) d u \\
L^{\prime}(x) & =\int_{x}^{+\infty} \frac{d N_{u}}{d_{u}} x \frac{1}{u} t\left(\frac{x}{u}\right) d u
\end{aligned}
$$

nous avons

$0=L^{\prime}(x)-L(x) \frac{N_{x}^{\prime}}{N_{x}}=\int_{x}^{+\infty} t\left(\frac{x}{u}\right) x \frac{1}{u}\left[N_{u}^{\prime}-\frac{x}{u} N_{u} \frac{N_{x}^{\prime}}{N_{x}}\right] d u$

Cette égalité est vraie pour toute fonction $t$.

Nous avons donc $\frac{N_{u}^{\prime}}{N_{u}}=\frac{x}{u} \frac{N_{x}^{\prime}}{N_{x}}=\frac{k}{u}$

$N_{u}$ suit donc bien une distribution de Pareto de paramètre $-k$.

\section{REMERCIEMENT}

Je remercie les deux correcteurs anonymes, grâce auxquels j'ai pu procéder à des rectifications d'importance. Mille et un remerciements à MARTINE MANAUD.

\section{BIBLIOGRAPHIE}

[1] Erwin Straub (1971) Estimation of the number of Excess Claims by means of the Credibility theory. ASTIN Bulletin V.

[2] Mette: Rytgaard (1989) Estimation of the Pareto Distribution, (XXI ${ }^{\mathrm{e}}$ Colloque Astin à New-York).

\section{Claude Huyghues-Beaufond}

Assurances Générales de France, 8-10 rue Villedo, F-75001 Paris. 